\title{
Ciclone Tropical Eloise em Moçambique: Aplicação da Teoria da Motivação de Proteção e Teoria estendida do Comportamento Planejado na análise de fatores determinantes na intenção de preparação para o enfrentamento
}

\author{
Tropical Cyclone Eloise in Mozambique: Application of the Theory of Protection Motivation and \\ Extended Theory of Planned Behavior in the analysis of determining factors in the intention to \\ prepare for the confrontation \\ Ciclón tropical Eloise en Mozambique: Aplicación de la Teoría de la Motivación Protectora y la \\ Teoría Extendida del Comportamiento Planificado en el análisis de factores determinantes en la \\ intención de prepararse para el enfrentamiento
}

Recebido: 31/10/2021 | Revisado: 08/11/2021 | Aceito: 16/11/2021 | Publicado: 25/11/2021

\author{
Nelson Mário Banga \\ ORCID: https://orcid.org/0000-0001-9917-4075 \\ Universidade Zambeze, Moçambique \\ E-mail: nlsnbanga@gmail.com \\ Calisto Manuel Máquina \\ ORCID: https://orcid.org/0000-0002-6749-3482 \\ Universidade Zambeze, Moçambique \\ E-mail: calisto.calmaqna.maquina@gmail.com \\ Elisa da Conceição José Maria \\ ORCID: https://orcid.org/0000-0003-3134-8061 \\ Universidade Rovuma, Moçambique \\ E-mail: elisajmaria@gmail.com \\ Eufrásio João Sozinho Nhongo \\ ORCID: https://orcid.org/0000-0002-5453-7845 \\ Universidade Zambeze, Moçambique \\ E-mail: eufrasionhongo@yahoo.com \\ Elias Welengane \\ ORCID: https://orcid.org/0000-0001-7841-6821 \\ Universidade Zambeze, Moçambique \\ E-mail: eliaswelengane@yahoo.com.br
}

\begin{abstract}
Resumo
Neste estudo, foi feita a integração das Teorias da Motivação de Proteção e do Comportamento Planejado, com objetivo de determinar os fatores que afetaram a intenção de preparação entre os residentes do Posto Administrativo de Macate na região centro de Moçambique, para enfrentar o ciclone tropical Eloise. Diversos fatores como a severidade percebida, vulnerabilidade percebida, controle comportamental percebido, norma subjetiva, atitude e intenção de preparação foram analisados utilizando a Modelagem de Equações Estruturais (Structural Equation Modeling-SEM). Os resultados demonstraram que as variáveis latentes atitude, vulnerabilidade percebida, controle comportamental percebido e normas subjetivas tiveram efeito direto significativo na intenção de preparação. $\mathrm{O}$ modelo estendido demostrou um melhor ajuste aos dados intenção de preparação para o enfrentamento dos efeitos dos ciclones.

Palavras-chave: Ciclone Tropical; Teoria da motivação de proteção; Teoria do comportamento planejado; Modelagem de equações estruturais.

Abstract

In this study, the Theories of Protection Motivation and Planned Behavior were integrated, in order to determine the factors that affected the intention of preparing among residents of the Administrative Post of Macate, in central Mozambique, to face the tropical cyclone Eloise. Several factors such as perceived severity, perceived vulnerability, perceived behavioral control, subjective norm, attitude and intention to prepare were analyzed using Structural Equation Modeling-SEM. The results showed that the latent variables attitude, perceived vulnerability, perceived behavioral control and subjective norms had a significant direct effect on the preparation intention. The extended model demonstrated a better fit to data preparedness intention to face the effects of the cyclones.
\end{abstract}


Keywords: Tropical Cyclone; Protection motivation Theory; Theory of planned behavior; Structural equation modeling.

\section{Resumen}

En este estudio se integraron las Teorías de Motivación de Protección y Comportamiento Planificado, con el fin de determinar los factores que incidieron en la intención de prepararse entre los residentes del Puesto Administrativo de Macate en el centro de Mozambique, para enfrentar el ciclón tropical Eloise. Se analizaron varios factores como la severidad percibida, la vulnerabilidad percibida, el control conductual percibido, la norma subjetiva, la actitud y la intención de prepararse utilizando el Modelo de Ecuación Estructural-SEM. Los resultados mostraron que las variables latentes actitud, vulnerabilidad percibida, control conductual percibido y normas subjetivas tuvieron un efecto directo significativo sobre la intención de preparación. El modelo extendido demostró un mejor ajuste a la intención de preparación de datos para enfrentar los efectos de los ciclones.

Palabras clave: Ciclón tropical; Teoría de la motivación de protección; Teoría del comportamiento planificado; Modelado de ecuaciones estructurales.

\section{Introdução}

Ciclones tropicais (CTs), como um dos riscos ambientais, continuam a causar danos ao longo do canal de Moçambique (Matyas e Silva, 2013; Fitchett e Grab, 2014). Em março de 2019 o ciclone Idai, causou um número substancial de danos e a perda de centenas de vidas humanas, instalações e infraestruturas em Moçambique, Zimbábue e Malawi (Warren, 2019; Salih et al., 2020; Kolstad, 2021). Além disso, foi caraterizado por chuvas torrenciais (mais de $200 \mathrm{~mm}$ em 24h) e ventos fortes (até $220 \mathrm{~km} / \mathrm{h}$ ), causando inundações generalizadas severas e mais de 600 mortes em Moçambique (Charrua et al., 2021).

A frequência do ciclone tropical em Moçambique, tende a aumentar, com o aumento dos efeitos das alterações climáticas (Hoque et al., 2018). Este aumento coloca a vida de muitos moçambicanos situados na região costeira em situação de vulnerabilidade aos efeitos do ciclone. Uma forma de redução dos riscos do ciclone, se baseia na definição de teorias de preparação adequedas para o seu enfrentamento.

As Teorias da Motivação de Proteção (Protection Motivation Theory- PMT) (Heidenreich et al., 2020) é uma teoria amplamente utilizada em diversos campos, como comportamentos de proteção em desastres (Heidenreich et al., 2020), em estudos para explicar o motivo pelo qual as pessoas escolhem ou não a se preparar para enfrentar os desastres (Becker et al., 2017; Vinnell et al., 2020). A Teoria do Comportamento Planejado (Theory of Planned Behavior -TPB) é um modelo que pode ser usado para prever o comportamento de um indivíduo em determinadas situações bem como para avaliar a preparação para catástrofres (LaMorte, 2018, Prasetyo et al., 2021), e Samah et al., 2019). A TPB inicia com fatores que inspiram as famílias a se prepararem, avança na forma de interação e mudanças de atitudes, e termina com decisões de preparação (Ajzen, 1991).

Neste estudo, foram integradas a PMT e TPB, com objetivo de determinar os fatores que afetaram a intenção de preparação entre os residentes do Posto Administrativo de Macate na região centro de Moçambique para o enfrentamento do ciclone tropical Eloise. Diversos fatores como a severidade percebida, vulnerabilidade percebida, controle comportamental percebido, norma subjetiva, atitude e intenção de preparação foram analisados utilizando a Modelagem de Equações Estruturais (Structural Equation Modeling-SEM).

\section{Desenvolvimento de Hipóteses e Modelo de Pesquisa}

A TPB de Ajzen (1991) é uma das teorias mais usadas em situações de emergência e de preparação para desastres naturais (Paek et al., 2010; Samah, 2019). O modelo proposto a partir da TPB, a intenção de se preparar para riscos decorrentes de desastres naturais é determinada pelo controle comportamental percebido, normas subjetivas e pela atitude. 
A Teoria da motivação de proteção (PMT) de acordo com com Rogers (1975), decorre da avaliação de ameaças quanto dos processos de avaliação de enfrentamento, foi muito usada na avaliação da ameaça, que consiste na vulnerabilidade percebida (o julgamento de que alguém sentirá que sua saúde está sendo ameaçada) e na gravidade percebida (a medida da percepção dos riscos à saúde de alguém se ele não adotar o serviço móvel de saúde). A avaliação de enfrentamento, que consiste na eficácia da resposta, auto eficácia e custo de resposta, avalia principalmente a resposta à situação. Estudos realizados por Garcia et al. (2018) mostraram que a vulnerabilidade percebida pode afetar a força de ação de um indivíduo no controle da situação. Assim, levantamos a hipótese de que.

H1: A vulnerabilidade percebida teve um efeito direto significativo sobre o controle comportamental percebido.

Estudos realizados por Coninck et al. (2020) mostraram existência de uma conexão entre a vulnerabilidade percebida e a atitude no concernente as medidas de segurança mais rígidas. A partir disso, a relação entre atitude e vulnerabilidade percebida é notavelmente significativa, pois ambas refletem a percepção de uma pessoa sobre a possibilidade de uma ameaça à saúde e ao bem-estar, como riscos geológicos. Considerando isso, foi formulada a seguinte hipótese.

H2: A vulnerabilidade percebida teve um efeito direto significativo na atitude de preparação para o enfrentamento do ciclone tropical Eloise.

A percepção da gravidade de um perigo está significativamente relacionada ao controle comportamental das pessoas (Wu, 2020). Yeung e Morris (2001) evidenciaram que a gravidade percebida é afetada por normas subjetivas, seguida de uma influência indireta na atitude e no comportamento das pessoas. Indo mais além, Zhao et al. (2018) mostraram que a gravidade percebida, juntamente com outros fatores possuem uma influência significativa sobre a atitude. Assim sendo, formulamos as seguintes hipóteses:

H3: A Severidade Percebida teve um efeito direto significativo no Controle Comportamental Percebido.

H4: A Severidade Percebida teve um efeito direto significativo na Atitude.

O controle comportamental percebido, definido como a facilidade ou dificuldade de realizar o comportamento (Ham et al., 2015 e Ataei et al., 2021). De acordo com Ataei et al. (2021), um indivíduo realiza um comportamento se perceber que as pessoas mais importantes para ele aprovam a execução do mesmo. O que significa que a facilidade ou dificuldade percebida por um indivíduo em realizar um determinado comportamento pode afetar a pressão social dada pelo mesmo indivíduo a outras pessoas na realização do mesmo comportamento. Deste modo foi formulada a seguinte hipótese.

H5: O controle comportamental percebido teve um efeito direto significativo sobre as normas subjetivas.

Alguns estudos sobre prevenção de desastres evidenciaram uma relação entre a atitude, crenças e valores (Chou et al., 2015). Aboelmaged (2021) sugeriu que embora uma atitude negativa em relação a um determinado sistema ou inovação, os indivíduos os adotam devido a pressões sociais. Indo mais além, Song e Shi (2020) afirmaram que quanto mais positiva for a atitude de um indivíduo maior será a pressão social externa para a adoção de comportamentos adaptáveis às alterações climáticas e aos riscos meteorológicos. Assim, foi formulada a seguinte hipótese.

H6: A atitude teve um efeito direto significativo na norma subjetiva.

A atitude em relação ao comportamento refere-se à percepção do indivíduo realizando um determinado comportamento. Estudos mostraram que existe uma correlação entre a atitude e a intenção de se preparar para enfrentar os 
desastres (Hoffman e Muttarak; 2017). Para Hoffman e Muttarak (2017), a atitude frente diante de eventos catastróficos pode estar relacionada à utilização de medidas preventivas. Sendo assim, foi formulada a seguinte a hipótese.

H7: A atitude teve um efeito direto significativo sobre a intenção de preparação para o enfrentamento do ciclone tropical Eloise.

A norma subjetiva é definida como as recompensas normativas, valores e desejos, cujo é expresso pela aprovação ou desaprovação dos outros (Ajzen, 1991). A maioria dos estudos sobre terremotos enfatizou o valor da preparação para terremotos (Kahlor et al., 2019; Doyle et al., 2020; Manandhar, 2016). No entanto, a intenção de preparação ou busca de informação ou apoio externo pode ser influenciada por normas subjetivas devido a esforços anteriores de busca (Kahlor et al., 2019). Desta forma, foi formulada a seguinte hipótese.

H8: As normas subjetivas tiveram um efeito direto significativo sobre a intenção de preparação para o enfrentamento do ciclone tropical Eloise.

O controle comportamental percebido representado pelas habilidades e competências de uma pessoa em lidar com uma determinada situação ou tarefa (Ham et al., 2015), tem um efeito positivo em relação à intenção de busca ou intenção de preparação (Kahlor et al., 2019). Portanto, foi formulada a seguinte hipótese:

H9: O controle comportamental percebido teve um efeito direto significativo sobre a intenção de preparação para o enfrentamento do ciclone tropical Eloise.

A Figura 1 representa o referencial teórico deste estudo. Apresenta a integração da teoria do comportamento planejado e a teoria da motivação da proteção.

Figura 1. Referencial teórico da pesquisa.

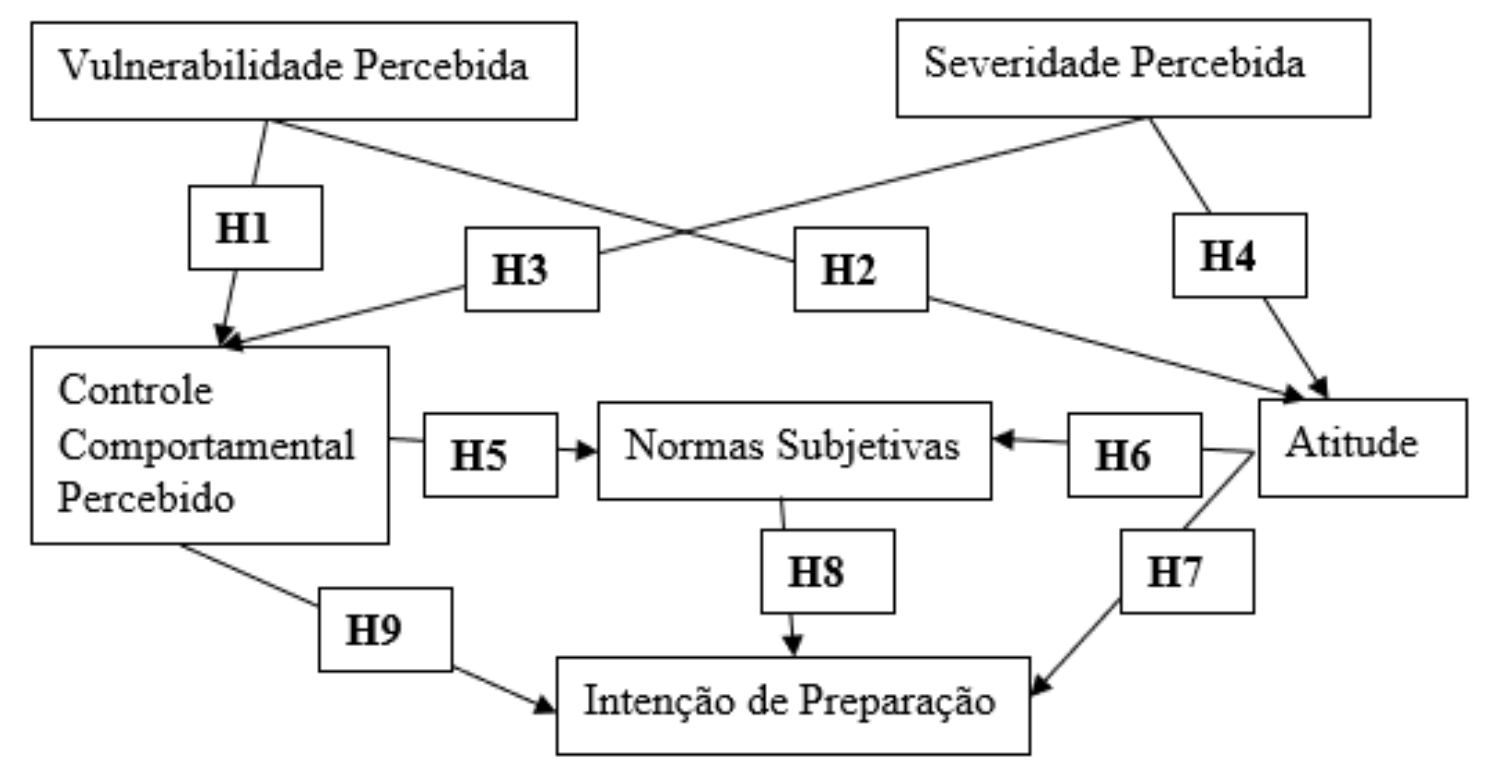

Fonte: Autores 


\section{Metodologia}

\subsection{Amostragem e coleta de dados}

O Posto Administrativo de Macate foi escolhido como um local de estudo de caso devido à ocorrência de dois grandes eventos de ciclones tropicais (Idai e Eloíse), que destruiu diversas culturas agrícolas, destruiu diversas residências, o que causou desabrigo de várias pessoas, e elevou o nível de necessidades de assistência direta em víveres e outros tipos de cuidados, das vítimas dos dois ciclones. A combinação da vulnerabilidade das populações e carência de infraestruturas de resilientes aos desastres naturais tornaram o Posto Administrativo de Macate, mais atrativo para a sua consideração neste estudo.

Os dados foram coletados usando um questionário aplicado em três bairros do Posto Administrativo de Macate, na região centro de Moçambique (Figura 2). Uma das técnicas utilizadas na determinação da amostra foi a utilização da fórmula de Slovin para determinar o número mínimo de amostras a 5\% de erro de estimativa (equação 1). De um universo de 22772 habitantes o número mínimo de amostra foi de 394 pessoas. A lista de perguntas usadas neste questionário, incluem itens relacionados com: (1) Informações demográficas (gênero idade, escolaridade, renda mensal e tipo de residência), (2) Vulnerabilidade percebida, (3) Severidade percebida, (4) Controle comportamental percebido, (5) norma subjetiva, (6) atitude e (7) intenção de preparação.

$$
n=\frac{\mathbb{N}}{1+\mathbb{N} e^{2}}
$$

Sendo $n$ o tamanho da amostra, $N$ tamanho da população e $e$ o erro de estimativa.

Figura 2. Localização geográfica da área de estudo em Moçambique.

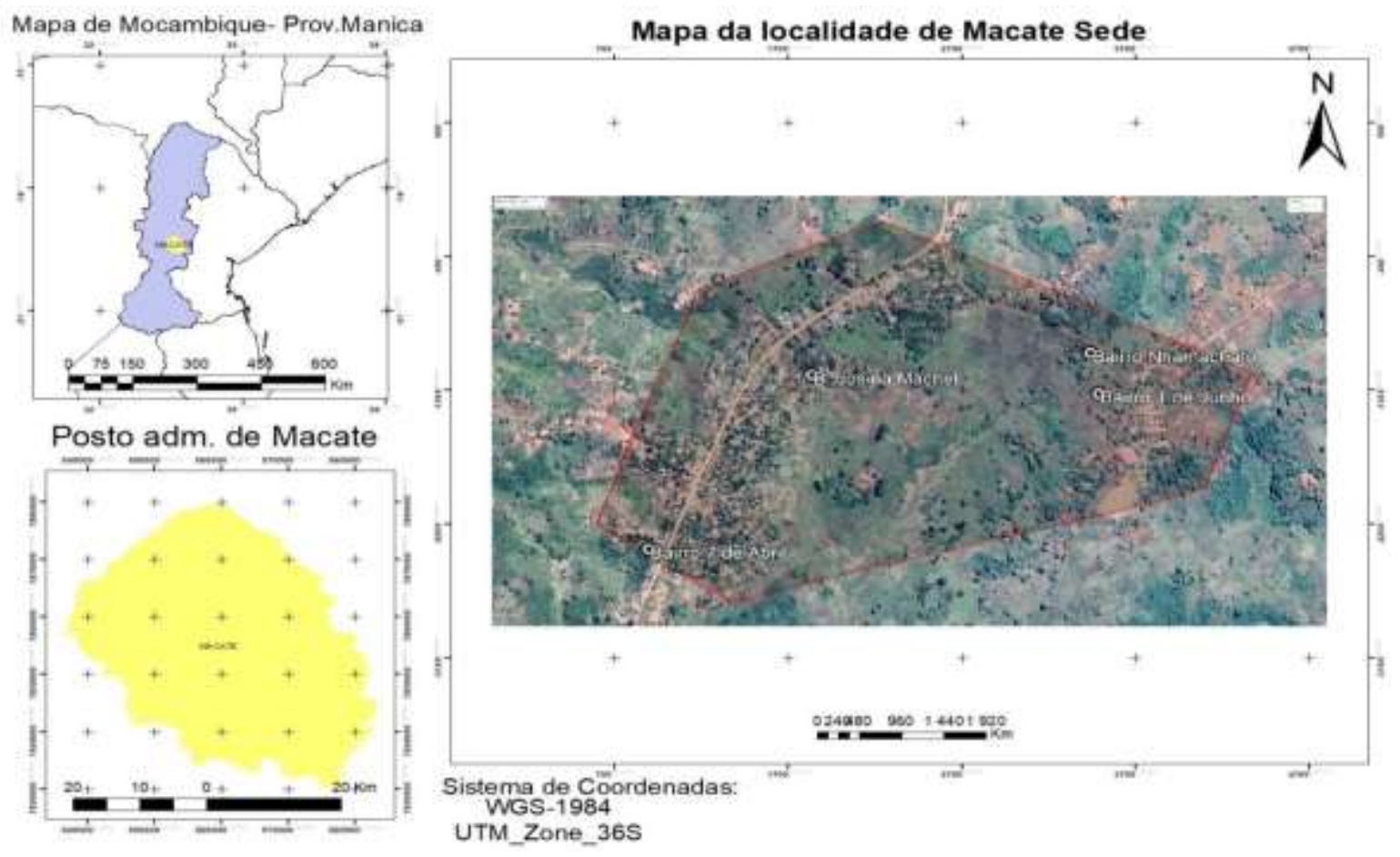

Fonte: Autores.

\subsection{Modelo de medição}

Foi utilizada uma abordagem quantitativa baseada no teste de um modelo de equações estruturais (SEM) para aferir as relações existentes entre as variáveis latentes (Vulnerabilidade percebida, Severidade percebida, Controle comportamental 
percebida, Norma subjetiva, Atitude e Intenção de preparação). A SEM é uma técnica de análise comumente usada para testar modelos em diferentes campos das ciências sociais e comportamentais (Ullman \& Bentler, 2012; Savari \& Gharechaee, 2020). Além disso, permite avaliar de forma simultânea a relação entre as variáveis independentes e uma ou variáveis dependentes, que podem ser observáveis ou não diretamente observáveis, como as variáveis latentes (Kiraz et al., (2020). A utilização desta técnica neste estudo deve-se ao fato dela permitir a verificação simultânea da causalidade entre as diversas variáveis.

No geral, haviam seis construtos, três intermediários (Controle comportamental percebido, norma subjetiva e atitude) e um construto dependente (intenção de preparação). Todos construtos latentes foram medidos utilizando uma escala Likert de 5 pontos (1- discordo completamente e 5- concordo completamente). A Tabela 1 descreve os itens usados para medir cada construto.

Tabela 1. Itens de medição de cada variável com base no TPB e PMT.

\begin{tabular}{|c|c|}
\hline Variáveis & Item \\
\hline \multirow[t]{2}{*}{$\begin{array}{l}\text { Vulnerabilidade } \\
\text { percebida (VP) }\end{array}$} & $\begin{array}{l}\text { Minha experiência passada com o ciclone tropical Idai me fez acreditar que } \\
\text { ficaríamos desabrigados/ desalojados em decorrência do ciclone tropical } \\
\text { Eloise. }\end{array}$ \\
\hline & $\begin{array}{l}\text { Baseado na ocorrência do ciclone tropical Idai o ciclone tropical Eloise, } \\
\text { destruiria infraestruturas entre outros bens. }\end{array}$ \\
\hline \multirow[t]{2}{*}{$\begin{array}{l}\text { Severidade percebida } \\
\text { (SP) }\end{array}$} & $\begin{array}{l}\text { Baseado na ocorrência da Tempestade Tropical Idai, a Tempestade Tropical } \\
\text { Eloise também causaria acidentes e perdas de vidas humanas. }\end{array}$ \\
\hline & $\begin{array}{l}\text { A Tempestade Tropical Eloise destruiria as culturas agrícolas, ameaçando } \\
\text { assim a segurança alimentar. }\end{array}$ \\
\hline \multirow[t]{2}{*}{ Atitude (AT) } & $\begin{array}{l}\text { Criei a segurança da minha família para evitar que se machucasse ou corresse } \\
\text { o risco de perda de vida. }\end{array}$ \\
\hline & Planifiquei todas ações que visavam à redução de riscos. \\
\hline \multirow[t]{2}{*}{ Norma subjetiva (NS) } & $\begin{array}{l}\text { A maior parte das pessoas que eu conheço armazenaram alimentos incluindo } \\
\text { água, entre outros kits de primeiros socorros. }\end{array}$ \\
\hline & $\begin{array}{l}\text { A maior parte das pessoas que conheço protegeram suas casas colocando } \\
\text { sacos de areia por cima dos tetos, bem como protetores sobre as janelas. }\end{array}$ \\
\hline \multirow{2}{*}{$\begin{array}{l}\text { Controle de } \\
\text { comportamento } \\
\text { percebido }(\mathrm{CCP})\end{array}$} & A segurança da minha família dependia inteiramente de mim. \\
\hline & $\begin{array}{l}\text { Dada a minha incapacidade de lidar com as consequências do ciclone tropical } \\
\text { Idai procurei ajuda de outras pessoas. }\end{array}$ \\
\hline \multirow{2}{*}{$\begin{array}{l}\text { Intenção de preparação } \\
\text { (IP) }\end{array}$} & Procurei por informações sobre medidas de proteção. \\
\hline & Identifiquei locais de abrigo \\
\hline
\end{tabular}

Fonte: Autores.

\subsection{Análise Estatística}

Considerando a complexidade do modelo, o SEM foi executado por meio do software R versão 3.6.3. Um estimador robusto de máxima verossimilhança, foi usado para controlar o efeito da falta de normalidade multivariada observada nos dados. Para testar a validade do modelo foi avaliado os valores de $\alpha$ de Cronbach, variância média extraída (AVE) e confiabilidade composta (CR). Além disso foi avaliado o ajuste do modelo, examinando o índice de ajuste geral $\chi^{2} / g 1$ (quiquadrado relativo), índice de ajuste comparativo (CFI), índice de Tucker Lewis (TLI), erro quadrático médio de aproximação (RMSEA), e raiz residual média padronizada (SRMR).

O estudo foi realizado recorrendo as metodológicas dos autores Koche (2011), Ludke e Andre (2013), Yin (2015), Estrela (2018) e Pereira et al. (2018). 


\section{Resultados e Discussão}

\subsection{Perfil dos entrevistados}

A Tabela 2 representa as estatísticas descritivas dos entrevistados. Entre os 394 entrevistados 50,3\% eram mulheres e 49,7\% homens. A maioria dos entrevistados tinha 30 - 39 anos (36\%), seguida de 40 - 49 (24,9\%), 20-29 (20,3\%), 50-59 $(15,5 \%)$ e $60-70(3,3 \%)$. Cerca de 44,2 \% dos entrevistados tinham o ensino secundário, $19,8 \%$ ensino primário do $1^{\circ}$ grau, $16,2 \%$ ensino primário do $2^{\circ}$ grau, $12,4 \%$ alfabetização, 5,3\% ensino técnico profissional e 2,0\% ensino superior. A maioria dos entrevistados tem uma renda mensal inferior a 4.000,00 MT (46,4\%) e apenas 2,0\% com tinha renda mensal superior a 12.000,00 MT.

Tabela 2. Estatística descritiva dos entrevistados $(n=394)$.

\begin{tabular}{|c|c|c|c|}
\hline Caraterísticas & Categoria & $\mathbf{N}$ & $\%$ \\
\hline \multirow[t]{2}{*}{ Género } & Masculino & 196 & 49,7 \\
\hline & Femenino & 198 & 50,3 \\
\hline \multirow[t]{5}{*}{ Idade } & $20-29$ & 80 & 20,3 \\
\hline & $30-39$ & 142 & 36,0 \\
\hline & $40-49$ & 98 & 24,9 \\
\hline & $50-59$ & 61 & 15,5 \\
\hline & $60-70$ & 13 & 3,3 \\
\hline \multirow[t]{6}{*}{ Escolaridade } & Alfabetização & 49 & 12,4 \\
\hline & $\begin{array}{l}\text { Ensino Primário do } 1^{\circ} \\
\text { Grau }\end{array}$ & 78 & 19,8 \\
\hline & $\begin{array}{l}\text { Ensino Primário do } 2^{\circ} \\
\text { Grau }\end{array}$ & 64 & 16,2 \\
\hline & Ensino Secundário & 174 & 44,2 \\
\hline & $\begin{array}{l}\text { Ensino Técnico } \\
\text { Profissional }\end{array}$ & 21 & 5,3 \\
\hline & Ensino Superior & 8 & 2,0 \\
\hline \multirow[t]{4}{*}{$\begin{array}{l}\text { Renda } \\
\text { (MT) }\end{array}$} & $<4.000,00$ & 183 & 46,5 \\
\hline & $4.000,00-8.000,00$ & 157 & 39,8 \\
\hline & $8.000,00-12.000,00$ & 46 & 11,7 \\
\hline & $>12.000,00$ & 8 & 2,0 \\
\hline \multirow[t]{4}{*}{ Residência } & Adobe & 262 & 66,5 \\
\hline & Alvenaria & 114 & 28,9 \\
\hline & Madeira - Zinco & 1 & 0,3 \\
\hline & Pau a Pique & 17 & 4,3 \\
\hline
\end{tabular}

Fonte: Autores.

\subsection{Modelo de medição TPB estendido}

Os resultados mostraram que o modelo em estudo apresenta um ajuste adequado, conforme os valores de referência (Gefen et al., 2000; Prasetyo et al., 2021; Steiger, 2007) (Tabela 3). As cargas fatoriais padronizadas ( $\beta$ ) na sua maioria foram estatisticamente significativas ao nível de erro de 5\% ( $\mathrm{p}<0,05)$. A Tabela 4 apresenta a confiabilidade composta por cada variável latente, os valores de $\alpha$ de Cronbach, variância média extraída (AVE) e confiabilidade composta (CR) estavam na sua maioria acima do intervalo mínimo aceitável, 0,7; 0,5 e 0,7 respectivamente (Alumran et al., 2014; Yang et al., 2020; Prasetyo et al., 2021). Assim, conclui-se que o modelo de mensuração proposto neste estudo demonstrou validade discriminante adequada. 
Tabela 3. Ajuste do modelo.

\begin{tabular}{lcc}
\hline Índice de ajuste & $\begin{array}{c}\text { Estimativas de } \\
\text { Parâmetros }\end{array}$ & Corte mínimo \\
\hline Índice de ajuste comparativo (CFI) & 0,957 & $>0,80$ \\
\hline Índice de Tucker Lewis (TLI) & 0,800 & $>0,80$ \\
\hline Erro de raiz quadrada média (RMSEA) & 0,067 & $<0,07$ \\
\hline Raiz quadrada média residual (SRMR) & 0,041 & $<0,06$ \\
\hline
\end{tabular}

Fonte: Autores.

Tabela 4. Análise de validade, fiabilidade e colinearidade do modelo de medição.

\begin{tabular}{cccc}
\hline & $\alpha$ de Cronbach & AVE & CR \\
\hline VP & 0,387 & 0,086 & 0,155 \\
\hline SP & 0,854 & 0,774 & 0,871 \\
\hline AT & 0,766 & 0,628 & 0,770 \\
\hline NS & 0,764 & 0,706 & 0,818 \\
\hline CCP & 0,866 & 1,126 & 1,072 \\
\hline IP & 0,742 & 0,588 & 0,741 \\
\hline
\end{tabular}

Fonte: Autores.

\subsection{Fatores que influenciaram a intenção de preparação para o enfrentamento do ciclone tropical Eloise.}

De acordo com o referencial teórico do estudo, os resultados mostram que o SEM se ajusta bem aos dados: $\chi^{2}(3)=$ $11,310(\mathrm{p}<0,05), \mathrm{RMSEA}=0,06(90 \%$ CI $[0,038 ; 0,146]), \mathrm{CFI}=0,95, \mathrm{SRMR}=0,04$. A maioria de nossas hipóteses foi comprovada ( $\mathrm{p}<0.05$; Figura 2), com exceção das relações entre vulnerabilidade percebida e o controle comportamental percebido (H1: a vulnerabilidade percebida tem um efeito significativo no controle comportamental percebido); severidade percebida e o controle comportamental percebido (H3:a severidade percebida tem um efeito significativo sobre o controle comportamental percebido); severidade percebida e atitude (H4: a severidade percebida tem um efeito significativo na atitude), e controle comportamental percebido e a norma subjetiva (H5: o controle comportamental percebido tem um efeito significativo nas norma subjetivas). No geral, as variáveis latentes controle comportamental percebido, norma subjetiva, atitude e vulnerabilidade percebida foram responsáveis por 36,7\% da variância da intenção de preparação para o enfrentamento do ciclone Eloise. 
Figura 3. Modelo de medição TPB estendido com coeficientes de trilha padronizados. * $\mathrm{p}<0,05, * * \mathrm{p}<0,01, * * * \mathrm{p}<0,001$, ' , indica não significativo.

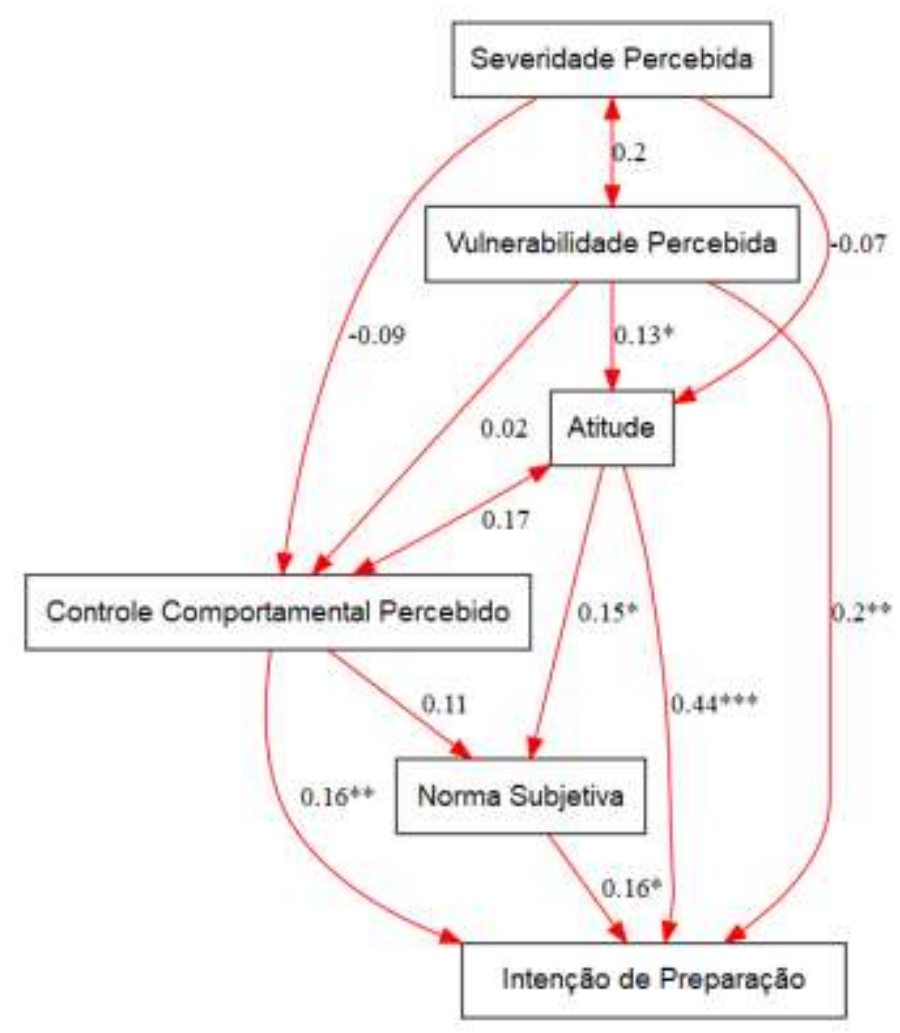

Fonte: Autores.

\subsection{Discussão}

O presente estudo integrou a Teoria de Motivação de Proteção (PMT) e a Teoria do Comportamento Planejado (TPB) para avaliar os fatores que determinaram a intenção de preparação para o enfrentamento da Tempestade Tropical Eloise em um Posto Administrativo da região centro de Moçambique. A modelagem de equações estruturais (SEM) foi utilizada para analisar a inter-relação entre a vulnerabilidade percebida, severidade percebida, atitude, norma subjetiva, controle comportamental percebido e a intenção de preparação.

A Modelagem de Equações Estruturais mostrou que a variável latente atitude teve um efeito positivo significativo em relação à intenção de preparação $(\beta=0,44 ; \mathrm{p}=0,000)$. Preocupar-se com a segurança da família e planificar-se com vista a reduzir os riscos bem como, assumir a responsabilidade de se preparar foram os indicadores significativos que motivaram à preparação para o enfrentamento. Esse resultado comprova o verificado em diversos estudos que indicaram a influência significativa da atitude nas intenções comportamentais das pessoas (Ataei et al., 2021; Aboelmaged, 2021; Savari e Gharechaee, 2020). Além disso, foi confirmado o efeito positivo e significativo da atitude sobre a norma subjetiva ( $\beta=0,15 ; \mathrm{p}$ $<0,05$ ), isso implica que a hipótese H6 (a atitude afeta diretamente a norma subjetiva) não foi rejeitada. Portanto, os resultados indicam que os entrevistados tiveram uma atitude positiva em relação a preparação para enfrentar o ciclone tropical, a medida que eles se preocuparam com sua segurança e adoptaram medidas protetivas em suas casas, bem como, asseguraram a estocagem de alimentos, água e alguns kits de primeiros socorros.

O controle comportamental percebido e a norma subjetiva tiveram um efeito significativo sobre a intenção de preparação pelos entrevistados $(\beta=0,16 ; \mathrm{p}<0,01)$ e $(\beta=0,16 ; \mathrm{p}<0,05)$, respetivamente. Isso implica que as hipóteses H8 e H9 (o controle comportamental percebido e a norma subjetiva têm efeito significativo na intenção de preparação para o 
enfrentamento) não foram rejeitadas. Ou seja, a dificuldade percebida (incapacidade de lidar com as consequências do ciclone) assim como a pressão social das estratégias de proteção das suas casas constituíram fatores que levaram aos entrevistados a se preparar para enfrentar o ciclone.

A severidade percebida não teve um efeito significativo em relação ao controle comportamental percebido $(\beta=-0,09$; $\mathrm{p}=0$,096). A hipótese H3 (a severidade percebida tem um efeito significativo sobre o controle comportamental percebido), foi rejeitada. A percepção da gravidade dos impactos do ciclone anterior que resultou na perda de vidas humanas e destruição de culturas agrícolas que afetou a segurança alimentar pode provavelmente não ter influenciado diretamente nas suas capacidades de lidar com o novo ciclone. Entretanto, a severidade percebida possui uma conexão indireta com o controle comportamental percebido (Yeung e Morris, 2001). Além disso, a severidade percebida não teve um efeito significativo na atitude de preparação ( $\beta=-0,07 ; \mathrm{p}=0,156$ ), o que sugere que a $\mathrm{H} 4$ (a severidade percebida tem um efeito significativo na atitude) foi rejeitada. No entanto, era de esperar que a percepção da gravidade dos danos do ciclone anterior sobre as vidas humanas tivesse um efeito direto sobre a atitude em relação aos danos no novo ciclone. Esse resultado contraria as evidências encontradas por $\mathrm{Wu}$ (2020; na qual se afirmou que a maneira como as pessoas percebem as coisas afeta sua atitude e comportamento em relação às coisas.

O controle comportamental percebido é um fator não significativo em relação às normas subjetivas $(\beta=0,11 ; \mathrm{p}=$ 0,053), sugerindo que a hipótese H5 (o controle comportamental percebido tem um efeito significativo nas normas subjetivas), foi rejeitada. Indicadores como garantir a segurança da família e buscar ajuda de outras pessoas para lidar com as consequências do ciclone afetaram positivamente a crença de as pessoas estarem a fazer a coisas certa para enfrentar o ciclone. Esse resultado está de acordo com o estudo de Song e Shi (2020); no qual afirmou que a adaptação de certos comportamentos afeta positivamente a formação de sua norma subjetiva.

A vulnerabilidade percebida teve um efeito positivo significativo em relação a atitude de preparação $(\beta=0,13 ; p<0,05)$ e um efeito positivo não significativo em relação ao controle comportamental percebido $(\beta=0,02 ; p=0,772)$. Estar ciente da magnitude de sua vulnerabilidade ajuda a tomar atitudes adequadas de preparação e consequentemente pode influenciar indiretamente as pessoas a estarem mais preparadas. O valor mais baixo de $(\beta=0,02)$ provavelmente pode ser que a vulnerabilidade ao risco ao novo ciclone não influencia necessariamente a necessidade das pessoas em buscar a ajuda de outros, e fortalecer sua capacidade de controlo da situação para enfrentar o novo ciclone tropical.

\section{Conclusão}

Moçambique é um país vulnerável às calamidades naturais, em um período de menos que dois anos foi assolado por três ciclones tropicais (Idai, Chalane e Eloise). A escassez de estudos sobre a preparação dos moçambicanos em relação às calamidades naturais, levou aos pesquisadores a abordarem esse problema. A pesquisa actual integrou a Teoria da Motivação de Proteção e a Teoria Estendida de Comportamento Planejado para avaliar os fatores que afetaram a intenção de preparação para o enfrentamento do ciclone tropical Eloise.

A modelagem de equações estruturais indicou que as variáveis latentes atitude $(\beta=0,44)$, vulnerabilidade percebida $(\beta=0,20)$, controle comportamental percebido $(\beta=0,16)$ e normas subjetivas $(\beta=0,16)$ tiveram efeito direto significativo na intenção de preparação, sendo que 36,7\% da variância da intenção de preparação para o enfrentamento do ciclone Eloise foi explicada por essas quatro variáveis latentes.

Embora as pessoas tivessem a percepção dos impactos causados pelo ciclone tropical anterior Idai, não foram suficientemente influenciados para tomar atitudes de preparação e muito menos impelidos a se capacitarem para enfrentarem um novo ciclone tropical. Assim sendo, recomenda-se que a melhoria na preparação para futuros ciclones se concentre mais na 
conscientização dos moçambicanos sobre a gravidade dos danos que os ciclones podem causar. Além disso, que eles estejam cientes sobre as atitudes que devem ser tomadas, pois afeta muito a forma como se deve preparar.

A utilização da teoria de comportamento planejado estendido neste estudo ofereceu um conhecimento do ponto de situação dos moçambicanos em relação a preparação e mitigação em relação aos ciclones que têm fustigado o país. Entretanto, este estudo pode ser usado como base para estudos futuros associados a outros desastres naturais, como por exemplo inundações e portanto, os resultados estudos podem ajudar na planificação de atividades de mitigação em relação a futuros ciclones.

\section{Referências}

Ajzen, I. (1991). The theory of planned behavior. Organizational behavior and human decision processes, 50(2), $179-211$.

Alumran, A., Hou, X. Y., Sun, J., Yousef, A. A., \& Hurst, C. (2014). Assessing the construct validity and reliability of the parental perception on antibiotics (PAPA) scales. BMC public health, 14(1), 1-9.

Artur, L., \& Hilhorst, D. (2012). Everyday realities of climate change adaptation in Mozambique. Global Environmental Change, 22(2), 529-536. http://doi.org/10.1016/j.gloenvcha.2011.11.013

Ash, K. D., \& Matyas, C. J. (2012). The influences of ENSO and the subtropical Indian Ocean Dipole on tropical cyclone trajectories in the southwestern Indian Ocean. International Journal of Climatology, 32(1), 41-56.

Ataei, P., Gholamrezai, S., Movahedi, R., \& Aliabadi, V. (2021). An analysis of farmers' intention to use green pesticides: The application of the extended theory of planned behavior and health belief model. Journal of Rural Studies, 81, 374-384.

Becker, J. S., Paton, D., Johnston, D. M., Ronan, K. R., \& McClure, J. (2017). The role of prior experience in informing and motivating earthquake preparedness. International journal of disaster risk reduction, 22, 179-193.

Budhathoki, N. K., Paton, D., Lassa, J. A., \& Zander, K. K. (2020). Assessing farmers' preparedness to cope with the impacts of multiple climate changerelated hazards in the Terai lowlands of Nepal. International Journal of Disaster Risk Reduction, 49, 101656.

Charrua, A. B., Padmanaban, R., Cabral, P., Bandeira, S., \& Romeiras, M. M. (2021). Impacts of the Tropical Cyclone Idai in Mozambique: A MultiTemporal Landsat Satellite Imagery Analysis. Remote Sensing, 13(2), 201.

Chou, J. S., Yang, K. H., \& Ren, T. C. (2015). Ex-post evaluation of preparedness education in disaster prevention, mitigation and response. International Journal of Disaster Risk Reduction, 12, 188-201.

De Coninck, D., d'Haenens, L., \& Matthijs, K. (2020). Perceived vulnerability to disease and attitudes towards public health measures: COVID-19 in Flanders, Belgium. Personality and Individual Differences, 166, 110220.

Doyle, E. E., McClure, J., Potter, S. H., Lindell, M. K., Becker, J. S., Fraser, S. A., \& Johnston, D. M. (2020). Interpretations of aftershock advice and probabilities after the 2013 Cook Strait earthquakes, Aotearoa New Zealand. International Journal of Disaster Risk Reduction, $49,101653$.

Estrela, C. (2018). Metodologia Científica: Ciência, Ensino, Pesquisa. Editora Artes Médicas.

Fitchett, J. M., \& Grab, S. W. (2014). A 66-year tropical cyclone record for south-east Africa: temporal trends in a global context. International Journal of Climatology, 34(13), 3604-3615.

Gabriel, E. H., Hoch, M. C., \& Cramer, R. J. (2019). Health Belief Model Scale and Theory of Planned Behavior Scale to assess attitudes and perceptions of injury prevention program participation: An exploratory factor analysis. Journal of science and medicine in sport, 22(5), 544-549.

Garcia, T. A., Fairlie, A. M., Litt, D. M., Waldron, K. A., \& Lewis, M. A. (2018). Perceived vulnerability moderates the relations between the use of protective behavioral strategies and alcohol use and consequences among high-risk young adults. Addictive behaviors, 81, $150-156$.

Gefen, D., Straub, D., \& Boudreau, M. C. (2000). Structural equation modeling and regression: Guidelines for research practice. Communications of the association for information systems, $4(1), 7$.

Ham, M., Jeger, M., \& Frajman Ivković, A. (2015). The role of subjective norms in forming the intention to purchase green food. Economic researchEkonomska istraživanja, 28(1), 738-748.

Heidenreich, A., Masson, T., \& Bamberg, S. (2020). Let's talk about flood risk-Evaluating a series of workshops on private flood protection. International Journal of Disaster Risk Reduction, 50, 101880.

Hoffmann, R., \& Muttarak, R. (2017). Learn from the past, prepare for the future: Impacts of education and experience on disaster preparedness in the Philippines and Thailand. World Development, 96, 32-51.

Hoque, M.-A.-A., Phinn, S., Roelfsema, C., \& Childs, I. (2018). Assessing tropical cyclone risks using geospatial techniques. Applied Geography, 98(2018), 22-33. https://doi.org/10.1016/j. apgeog.2018.07.004

https://cancercontrol.cancer.gov/brp/research/constructs/perceived-severity (2020) 
Janmaimool, P. (2017). Application of protection motivation theory to investigate sustainable waste management behaviors. Sustainability, 9(7), 1079.

Kahlor, L. A., Wang, W., Olson, H. C., Li, X., \& Markman, A. B. (2019). Public perceptions and information seeking intentions related to seismicity in five Texas communities. International journal of disaster risk reduction, 37, 101147.

Kiraz, A., Canpolat, O., Özkurt, C., \& Taşkın, H. (2020). Analysis of the factors affecting the Industry 4.0 tendency with the structural equation model and an application. Computers \& Industrial Engineering, 150, 106911.

Koche, J. C. (2011). Fundamentos de metodologia científica. Petrópolis: Vozes. http://www.brunovivas.com/wp-content/uploads/sites/10/2018/07 /K\%C3\%B6che-Jos\%C3\%A9-Carlos0D0AFundamentos-de-metodologia-cient\%C3\%ADfica-_teoria-da0D0Aci\%C3\%AAncia-e-inicia\%C3\%A7\%C3\%A3o$\%$ C3\%A0-pesquisa.pdfhttps://repositorio.ufsm.br/bitstream/handle/1/15824/Lic_Computacao_Metodologia-Pesquisa-Cientifica.pdf?sequence=1.

Kolstad, E. W. (2021). Prediction and precursors of Idai and 38 other tropical cyclones and storms in the Mozambique Channel. Quarterly Journal of the Royal Meteorological Society, 147(734), 45-57.

Laato, S., Islam, A. N., \& Laine, T. H. (2020). Did location-based games motivate players to socialize during COVID-19?. Telematics and Informatics, 54, 101458.

LaMorte, W. W. (2018). Behavioral change models: The theory of planned behavior. Retrieved November, 7, 2018.

Lazarus, R. S., \& Folkman, S. (1984). Stress, appraisal, and coping. Springer publishing company.

Lin, J., Zhu, R., Li, N., \& Becerik-Gerber, B. (2020). How occupants respond to building emergencies: A systematic review of behavioral characteristics and behavioral theories. Safety science, 122, 104540.

Ludke, M. \& Andre, M. E. D. A. (2013). Pesquisas em educação: uma abordagem qualitativa. E.P.U.

Manandhar, B. (2016). Remittance and earthquake preparedness. International Journal of Disaster Risk Reduction, $15,52-60$.

McCaughey, J. W., Mundir, I., Daly, P., Mahdi, S., \& Patt, A. (2017). Trust and distrust of tsunami vertical evacuation buildings: Extending protection motivation theory to examine choices under social influence. International journal of disaster risk reduction, 24, $462-473$.

Miles, A., Aboelmaged, M. (2021). E-waste recycling behaviour: An integration of recycling habits into the theory of planned behaviour. Journal of Cleaner Production, 278, 124182 .

Paek, H. J., Hilyard, K., Freimuth, V., Barge, J. K., \& Mindlin, M. (2010). Theory-based approaches to understanding public emergency preparedness: implications for effective health and risk communication. Journal of health communication, 15(4), 428-444.

Paul, B. K., \& Bhuiyan, R. H. (2010). Urban earthquake hazard: perceived seismic risk and preparedness in Dhaka City, Bangladesh. Disasters, 34(2), 337359.

Pereira A. S., Shitsuka, D. M., Parreira, FJ. \& Shitsuka, R (2018). Metodologia da pesquisa científica. UFSM.

Prasetyo, Y. T., Tanto, H., Mariyanto, M., Hanjaya, C., Young, M. N., Persada, S. F., ... \& Redi, A. A. N. P. (2021). Factors affecting customer satisfaction and loyalty in online food delivery service during the covid-19 pandemic: Its relation with open innovation. Journal of Open Innovation: Technology, Market, and Complexity, 7(1), 76.

Quan, L., Zhen, R., Yao, B., Zhou, X., \& Yu, D. (2017). The role of perceived severity of disaster, rumination, and trait resilience in the relationship between rainstorm-related experiences and PTSD amongst chinese adolescents following rainstorm disasters. Archives of psychiatric nursing, 31 (5), 507-515.

Rogers, R. W. (1975). A protection motivation theory of fear appeals and attitude change1. The journal of psychology, 91(1), 93-114.

Salih, A. A., Baraibar, M., Mwangi, K. K., \& Artan, G. (2020). Climate change and locust outbreak in East Africa. Nature Climate Change, 10(7), 584-585.

Samah, A. A., Zaremohzzabieh, Z., Shaffril, H. A. M., D'Silva, J. L., \& Kamarudin, S. (2019). Researching natural disaster preparedness through health behavioral change models. Am J Disaster Med, 14(1), 51-63.

Samah, A. A., Zaremohzzabieh, Z., Shaffril, H. A. M., D'Silva, J. L., \& Kamarudin, S. (2019). Researching natural disaster preparedness through health behavioral change models. Am J Disaster Med, 14(1), 51-63.

Savari, M., \& Gharechaee, H. (2020). Application of the extended theory of planned behavior to predict Iranian farmers' intention for safe use of chemical fertilizers. Journal of Cleaner Production, 263, 121512.

Smelser, N. J., \& Baltes, P. B. (Eds.). (2001). International encyclopedia of the social \& behavioral sciences (Vol. 11). Amsterdam: Elsevier.

Song, Z., \& Shi, X. (2020). Cherry growers' perceived adaption efficacy to climate change and meteorological hazards in northwest China. International Journal of Disaster Risk Reduction, 46, 101620.

Song, Z., \& Shi, X. (2020). Cherry growers' perceived adaption efficacy to climate change and meteorological hazards in northwest China. International Journal of Disaster Risk Reduction, 46, 101620.

Steiger, J. H. (2007). Understanding the limitations of global fit assessment in structural equation modeling. Personality and Individual differences, 42(5), 893-898. 
Research, Society and Development, v. 10, n. 15, e247101522592, 2021

(CC BY 4.0) | ISSN 2525-3409 | DOI: http://dx.doi.org/10.33448/rsd-v10i15.22592

Ullman, J. B., \& Bentler, P. M. (2012). Structural equation modeling. Handbook of Psychology, Second Edition, 2.

Vinnell, L. J., Wallis, A., Becker, J. S., \& Johnston, D. M. (2020). Evaluating the ShakeOut drill in Aotearoa/New Zealand: effects on knowledge, attitudes, and behaviour. International Journal of Disaster Risk Reduction, 48, 101721.

Wang, X., Yuen, K. F., Shi, W., \& Ma, F. (2020). The determinants of passengers' safety behaviour on public transport. Journal of Transport \& Health, 18, 100905.

Warren, M. (2019). Why Cyclone Idai is one of the Southern Hemisphere's most devastating storms. Nature.

Wu, D. (2020). Empirical study of knowledge withholding in cyberspace: Integrating protection motivation theory and theory of reasoned behavior. Computers in Human Behavior, 105, 106229.

Wu, D. (2020). Empirical study of knowledge withholding in cyberspace: Integrating protection motivation theory and theory of reasoned behavior. Computers in Human Behavior, 105, 106229.

Yang, F., Tan, J., \& Peng, L. (2020). The effect of risk perception on the willingness to purchase hazard insurance-A case study in the Three Gorges Reservoir region, China. International Journal of Disaster Risk Reduction, 45, 101379.

Yeung, R. M., \& Morris, J. (2001). Food safety risk: Consumer perception and purchase behaviour. British food journal.

Yeung, R. M., \& Morris, J. (2001). Food safety risk: Consumer perception and purchase behaviour. British food journal.

Yin, R. K. (2015). O estudo de caso. Bookman.

Zhao, Y., Ni, Q., \& Zhou, R. (2018). What factors influence the mobile health service adoption? A meta-analysis and the moderating role of age. International Journal of Information Management, 43, 342-350. 